\title{
Sensitivity of MEG and EEG to Source Orientation
}

\author{
Seppo P. Ahlfors · Jooman Han $\cdot$ John W. Belliveau • \\ Matti S. Hämäläinen
}

Received: 19 May 2010/ Accepted: 2 July 2010/Published online: 18 July 2010

(C) The Author(s) 2010. This article is published with open access at Springerlink.com

\begin{abstract}
An important difference between magnetoencephalography (MEG) and electroencephalography (EEG) is that MEG is insensitive to radially oriented sources. We quantified computationally the dependency of MEG and EEG on the source orientation using a forward model with realistic tissue boundaries. Similar to the simpler case of a spherical head model, in which MEG cannot see radial sources at all, for most cortical locations there was a source orientation to which MEG was insensitive. The median value for the ratio of the signal magnitude for the source orientation of the lowest and the highest sensitivity was 0.06 for MEG and 0.63 for EEG. The difference in the sensitivity to the source orientation is expected to contribute to systematic differences in the signal-to-noise ratio between MEG and EEG.
\end{abstract}

Keywords Magnetoencephalography ·

Electroencephalography $\cdot$ Suppression ratio ·

Current dipole $\cdot$ Radial source current

\section{Introduction}

One of the main differences between magnetoencephalography (MEG) and electroencephalography (EEG) is

S. P. Ahlfors $(\square) \cdot$ J. Han · J. W. Belliveau · M. S. Hämäläinen MGH/HST Athinoula A. Martinos Center for Biomedical Imaging, Massachusetts General Hospital/Harvard Medical School, 149 13th Street, Rm 2301, Charlestown, MA 02129, USA

e-mail: seppo@nmr.mgh.harvard.edu

S. P. Ahlfors · J. W. Belliveau · M. S. Hämäläinen Harvard-MIT Division of Health Sciences and Technology, Cambridge, MA 02135, USA thought to be that MEG is mainly sensitive to tangentially oriented sources, whereas EEG can detect sources of all orientations (Baillet et al. 2001; Cohen and Cuffin 1991; Hämäläinen et al. 1993). Theoretically, a radially oriented current dipole produces no magnetic field outside a spherically symmetric volume conductor (Baule and McFee 1965; Grynszpan and Geselowitz 1973). However, since the human head is not exactly spherically symmetric, the radial orientation is not well defined, and an approximately radial source in the brain is not necessarily silent in MEG (Cuffin 1990). Therefore, it is possible that all source orientations generate a magnetic field that is detectable by MEG.

The relative magnitude of the MEG signal generated by a radial and a tangential source at the same location was examined experimentally by Melcher and Cohen (1988), who found that the signals differed by a factor of about 6 for several locations in the rabbit brain. Similar values were found in simulations taking into account realistic tissue properties of the human head (Haueisen et al. 1995). Only a small number of source locations, however, was examined in the previous studies. In the present simulation study we mapped the dependency of MEG and EEG signal magnitude on the orientation of a current dipole in a dense grid of source locations in the human cerebral cortex. We examined the ratio of the signals due to a dipole having an orientation with the lowest vs. the highest sensitivity for an array of MEG or EEG sensors. A forward solution for each source location was constructed using a boundary-element method. The source orientations with the lowest and the highest sensitivity were determined with the help of the singular value decomposition (SVD). Our goal was to confirm the existence of an insensitive source orientation for MEG at any given location in the human brain. 


\section{Materials and Methods}

The MEG and EEG forward models were based on anatomical data from a 42 -year old male volunteer subject. The procedures were approved by the Massachusetts General Hospital Institutional Review Board. High-resolution structural T1-weighted magnetic resonance images (MRIs) were acquired on a $1.5 \mathrm{~T}$ Siemens scanner $(\mathrm{TR}=2530 \mathrm{~ms}$, $\mathrm{TE}=3.25 \mathrm{~ms}$, flip angle $=7^{\circ}, 128$ sagittal slices, slice thickness $=1.3 \mathrm{~mm}$, voxel size $=1.3 \times 1.0 \times 1.3 \mathrm{~mm}^{3}$ ). A surface mesh of the gray-white matter border of the cerebral cortex with about 300000 vertices was constructed from the MRI using the Free Surfer software (Dale et al. 1999; Fischl et al. 1999). Each vertex represented a location of an MEG and EEG source.

The conductivity geometry of the head was modeled using a boundary element method (BEM) with three compartments: the brain, the skull, and the scalp (Hämäläinen and Sarvas 1989). The boundaries were determined from the MRI by identifying the inner and outer surfaces of the skull and the outer surface of the skin (Liu et al. 2002). Each surface consisted of 5120 vertices. The linear collocation method was used in the BEM calculations (Mosher et al. 1999). The conductivity ratios of 1:0.0125:1 were assumed for brain:skull:scalp (Geddes and Baker 1967).

The $N \times 3 M$-dimensional forward matrix A describes the relationship between the MEG and EEG signals in an array of $N$ sensors and the three components of a dipole moment vector at $M$ locations in the brain. Here $M$ was about 300,000. The MEG sensor array consisted of $N=102$ MEG magnetometers, measuring the magnetic field component approximately radial to the surface of the head; the EEG sensor array comprised $N=60$ scalp electrodes. The column vectors $\mathbf{a}_{j}$ of $\mathbf{A}$ correspond to the signal patterns generated by a single source element of unit amplitude. For EEG, the average reference was used. The forward matrix was computed using the MNE software (http://www.nmr.mgh.harvard.edu/martinos/userInfo/data/ sofMNE.php).

To examine the sensitivity of the MEG and EEG sensor arrays to sources of different orientations, we computed the SVD of the $N \times 3$-dimensional dipolar gain matrix $\mathbf{A}_{k}=\left[\mathbf{a}_{3(k-1)+1}, \mathbf{a}_{3(k-1)+2}, \mathbf{a}_{3(k-1)+3}\right]$ at each location indexed by $k=1, \ldots, M$ (Huang et al. 2007):

$\mathbf{A}_{k}=\sum_{j=1}^{3} \lambda_{k, j} \mathbf{u}_{k, j} \mathbf{v}_{k, j}^{\mathrm{T}}=\sum_{j=1}^{3} \mathbf{g}_{k, j} \mathbf{v}_{k, j}^{\mathrm{T}}$,

where $\mathbf{u}_{k, j}$ and $\mathbf{v}_{k, j}$ are the left and right singular vectors, respectively, and $\lambda_{k, 1}, \lambda_{k, 2}$, and $\lambda_{k, 3}$ are the singular values. The $N$-dimensional vector $\mathbf{g}_{k, j}=\lambda_{k, j} \mathbf{u}_{k, j}$ describes the signal pattern in the MEG or EEG sensor array generated by a current dipole of unit amplitude in the orientation $\mathbf{v}_{k, j}$. We denote the largest and smallest singular values, corresponding to the dipole orientation to which the MEG sensor array is the most and the least sensitive, as $\lambda_{k, 1}=\lambda_{k, \text { max }}$ and $\lambda_{k, 3}=\lambda_{k, \text { min }}$, respectively. In the spherically symmetric volume conductor model $\lambda_{k, \min }=0$ for MEG for all source locations, because the radial source component does not generate any MEG signals.

The ratio

$R_{\lambda}=\lambda_{k, \min } / \lambda_{k, \max }=\left\|\mathbf{g}_{k, \text { min }}\right\| /\left\|\mathbf{g}_{k, \text { max }}\right\|$

was computed for all the locations on the cortical surface. This measure of relative sensitivity to the "suppressed" source orientation is analogous to the suppression ratio for radial vs. tangential dipoles studied previously (Haueisen et al. 1995; Melcher and Cohen 1988). Melcher and Cohen used the maximum signal power among pairs of planar gradiometers, whereas our definition is similar to the $R_{p}$ measure of Haueisen et al., which is based on the signal power in an array of sensors. Different definitions of the suppression ratio, however, have been reported to provide converging results (Haueisen et al. 1995). We note that instead of defining the "radial" and "tangential" orientations on the basis of a sphere fit on the local curvature of the inner surface of skull near the source location (Haueisen et al. 1995), the SVD automatically provides the source orientation of lowest and highest signal power in the sensor array.

\section{Results}

Distributions of the ratio $R_{\lambda}$ across the cerebral cortex are shown in Fig. 1. For MEG, the median value of $R_{\lambda}$ was 0.06 and $95 \%$ of the values were below 0.23 . The prominence of small values for $R_{\lambda}$ for MEG suggests that for most locations on the cortex there was a source orientation with which little if any MEG signals was generated. In contrast, for EEG the median value of $R_{\lambda}$ was 0.63 and $95 \%$ of the values were above 0.42 .

For superficial regions of the lateral surface of the temporal, parietal, and occipital cortices, the ratio $R_{\lambda}$ for MEG was typically small, indicating the presence of a source orientation to which MEG is insensitive to. The largest values of the ratio $R_{\lambda}$ for MEG were found in the orbitofrontal regions and sulcal regions of the lateral frontal cortex, as well as in the Sylvian fissure including the insula, and the medial surface. Occasional isolated vertices with large values of $R_{\lambda}$ were seen at the crowns of gyri; these are likely to be caused by numerical inaccuracy of the forward model for sources that are located close to the inner skull boundary. For EEG, the smallest values for $R_{\lambda}(\sim 0.2)$ were found in the orbitofrontal and temporal 

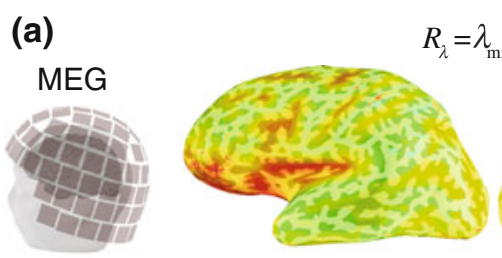

$R_{\lambda}=\lambda_{\min } / \lambda_{\max }$
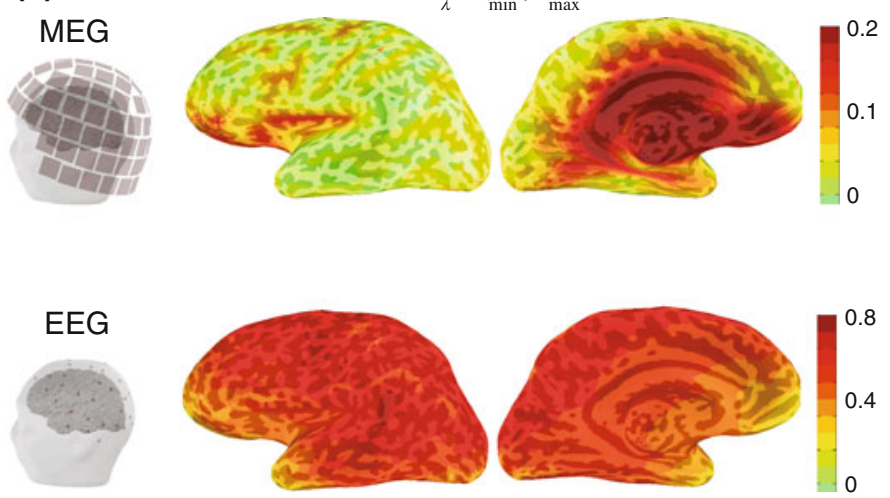

(b) $n_{\mathrm{MEG}}$
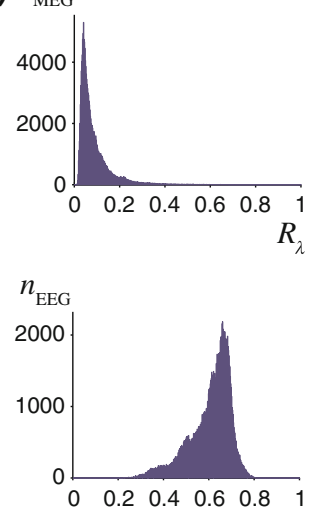

Fig. 1 Distribution of $R_{\lambda}$, measuring the relative sensitivity to sources of different orientation and calculated as the ratio of the smallest and largest singular values of the dipole gain matrix for MEG (top) and EEG (bottom). $\boldsymbol{a}$ Spatial maps of $R_{\lambda}$ for the left hemisphere are shown in a lateral and medial view of an inflated representation of the cerebral cortex. The curvature of the cortex is indicated by darker (sulci) and lighter (gyri) regions through the semi-transparent color-

pole regions, likely reflecting a limited coverage of the EEG scalp electrode array in the face and temple areas. Also for MEG, the results for the orbitofrontal region are likely to be affected by the positioning of the sensor array (Marinkovic et al. 2004).

The sensitivity of MEG and EEG for three orthogonal source orientations at each location on the cortex is shown in Fig. 2. The sensitivity was quantified by the three singular values of the dipole gain matrix $\mathbf{A}_{k}$. The singular value corresponding to the highest sensitivity $\left(\lambda_{k, \max }\right)$ showed a strong depth dependency for MEG. The spatial distribution of the smallest singular value $\left(\lambda_{k, \min }\right)$ for MEG was similar to that of the ratio $R_{\lambda}$ shown in Fig. 1, except that the large values of $R_{\lambda}$ for MEG occurred in the

(a) MEG

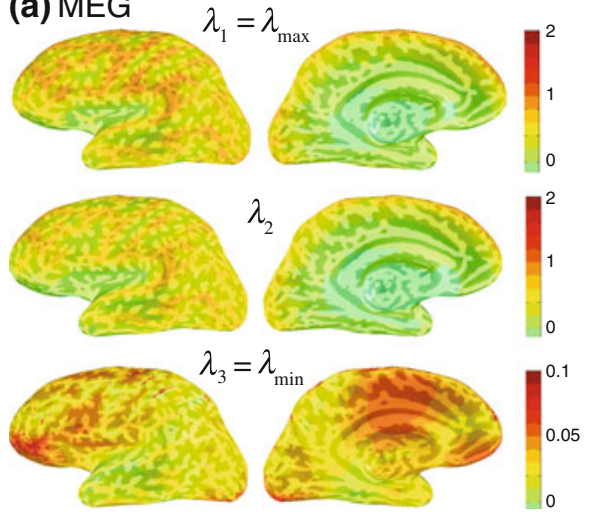

coded map of $R_{\lambda}$. Note the different color scales for MEG and EEG. The location of the MEG sensors and EEG electrodes with respect to the cortical surface and the scalp are shown on the left. $\boldsymbol{b}$ Histograms of the $R_{\lambda}$ values across all locations on the cortex, showing the number of vertices for MEG and EEG $\left(n_{\mathrm{MEG}}, n_{\mathrm{EEG}}\right)$ with a given value of $R_{\lambda}$

subcortical regions near the center of the head where $\lambda_{k, \max }$ was very small.

The histograms in Fig. 2a illustrate how the sensitivity of MEG for one source orientation (corresponding to $\left.\lambda_{k, \min }\right)$ is typically much smaller than the other orientations. For EEG, the histograms indicate that there is no orientation for which the sensitivity is very small (Fig. 2b). Furthermore, for MEG the distributions of the two largest singular values $\left(\lambda_{k, 1}\right.$ and $\left.\lambda_{k, 2}\right)$ were similar, whereas for EEG the distribution of the two smallest singular values ( $\lambda_{k, 2}$ and $\lambda_{k, 3}$ ) were similar. The two similar distributions in each case are likely to be mainly due to tangentially oriented sources, which generated similar signal patters rotated by $90^{\circ}$ with respect to each other, whereas a radial
Fig. 2 Distribution of the sensitivity to three orthogonal source components at each location on the cortex, indicated by the singular values $\left(\lambda_{k, 1}=\lambda_{k, \max }, \lambda_{k, 2}, \lambda_{k, 3}=\lambda_{k, \min }\right)$ of the dipole gain matrix $\mathbf{A}_{k}$ for MEG (a) and EEG (b). Note that for MEG, the color scales in the (b) EEG
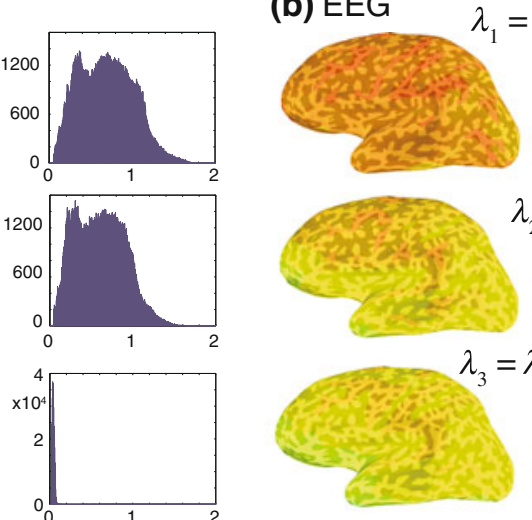

$\lambda_{1}=\lambda_{\max }$
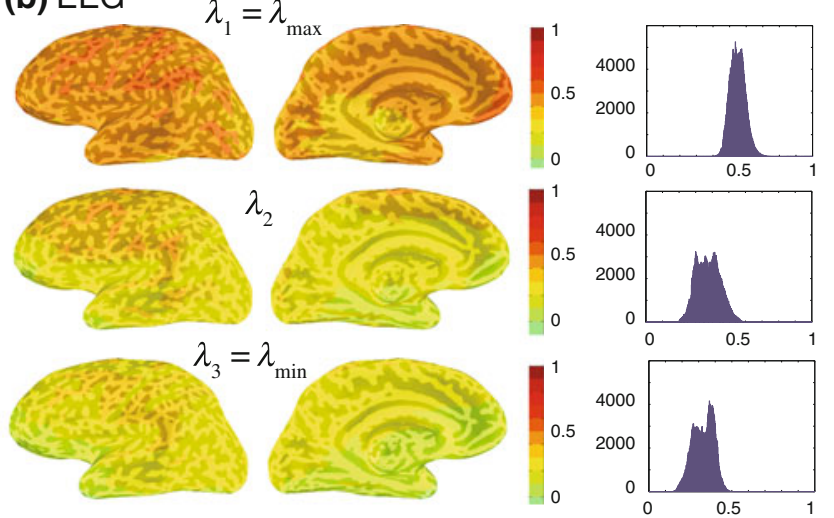

spatial map and the vertical scales in the histogram are different for the singular value for the orientation of the lowest sensitivity $\left(\lambda_{k, 3}\right)$ from those for $\lambda_{k, 1}$ and $\lambda_{k, 2}$. The units for $\lambda_{k, i}$ are pT/nAm for MEG and $\mu \mathrm{V} / \mathrm{nAm}$ for EEG 
source generates a signal pattern that is qualitatively different from that from the tangential sources.

\section{Discussion}

To examine the dependency of MEG and EEG signals on the source orientation, we compared the forward solutions for current dipoles with the orientation of the lowest and the highest sensitivity across the cerebral cortex, i.e., dipole orientations generating the smallest and the largest signals. For most locations, especially the superficial ones, there was a "quiet" source orientation for MEG, analogously to the radial orientation in the spherical model. For EEG, the sensitivity depended on the source orientation much less than for MEG.

The mean value for the sensitivity ratio $R_{\lambda}$ for MEG $(0.06 \approx 1 / 17)$ was somewhat smaller than the experimental value (1/6) observed in the rabbit brain (Melcher and Cohen 1988). This difference is not surprising, given the difficulty of aligning an artificial current dipole exactly along the orientation of the lowest sensitivity. The SVD analysis provided the orientation of the lowest sensitivity based on the signal power in the array of sensors, rather than relying on the "radial" orientation defined in terms of the local curvature of the skull (Haueisen et al. 1995; Melcher and Cohen 1988).

In some regions, sources with the orientation of lowest MEG sensitivity generated substantial MEG signals, up to about $20 \%$ of that generated by a source with the orientation of highest MEG sensitivity at the same location. A region with a non-vanishing $R_{\lambda}$ for MEG implies that the spherically symmetric volume conductor model is not accurate for that region, warranting the use a more realistic head model in inverse modeling also for MEG. This is particularly important for deep cortical and subcortical regions (Parkkonen et al. 2009; Tesche et al. 1996). Further anatomical information about conductivity properties of the head could be taken into account by using finite element modeling (FEM) (Haueisen et al. 1995; Haueisen et al. 2002; Ramon et al. 2004; Wolters et al. 2006). For example, incorporating the cerebrospinal fluid or anisotropic conductivity in the white matter could affect the sensitivity measures examined in the present study. The regions where the deviations from the spherical model were largest, i.e., in frontal, insular, and medial regions, are in accordance with those found in previous studies comparing dipole localization accuracy in spherically symmetric volume conductor model vs. anatomically more realistic head models (Hämäläinen and Sarvas 1989; Haueisen et al. 2002; Schimpf et al. 2002; Tarkiainen et al. 2003; Wolters et al. 2006). We note that, even though $R_{\lambda}$ being close to zero does not guarantee that the spherical model will provide an accurate MEG forward solution for those locations, previous studies have suggested that often the spherical model does indeed provide an accurate forward model for MEG, especially for most of the superficial cortical regions (Huang et al. 1999).

Some caution is necessary in interpreting our results, as numerical accuracy of the forward model for sources close to the skull may suffer from the sparseness of the BEM vertices (Fuchs et al. 2001). Previous studies have suggested that the resolution used here provides a reasonable accuracy for most cortical source locations (Yvert et al. 1996). Furthermore, we selected the inner surface of the cerebral cortex, rather than, e.g., the middle of the gray matter, for the location of the sources, to avoid them being very close to the brain-skull interface when computing the forward model. Despite these precautions, we observed large values of $\lambda_{k, \text { min }}$ at some isolated superficial vertices, which presumably were caused by numerical instabilities.

Silent source elements are a special case of silent source distributions defined by the null space of the forward matrix (Mosher et al. 1999; Riera et al. 2006). Extensive coverage of the scalp by the sensor arrays, as well as combined use of EEG and MEG sensors help to minimize the null space. In general, the orientation of the source has a strong influence on the spatial pattern of the EEG and MEG signals. The importance of the source orientation for the interpretation of the data is sometimes overlooked in the common practice of visualizing only the estimated source magnitudes of inverse solutions.

Overall, the present results agree with the commonly held view that an important difference between MEG and EEG is that MEG is mainly sensitive to the tangential source components only, whereas EEG is sensitive to all components. Few cortical sources, however, are expected to have exactly the orientation of the lowest sensitivity in MEG (Hillebrand and Barnes 2002). Therefore, the depth, rather than the orientation, is likely to be the critical factor determining the detectability of an individual current dipole source in MEG. In other words, the sensitivity of MEG to source having the orientation corresponding to $\lambda_{k, \text { min }}$ is low at all cortical locations, whereas the sensitivity to the other two orientations depends strongly on the depth (Fig. 2). This is consistent with MEG being most sensitive to superficial sources, and the sensitivity being much reduced for deep sources (Cohen and Cuffin 1983; de Jongh et al. 2005; Goldenholz et al. 2009; Hillebrand and Barnes 2002).

The insensitivity of MEG to one source orientation has a specific consequence for extended patches of cortical activity. For extended sources, selective cancellation of MEG and EEG signals generated by tangential sources may occur (Ahlfors et al. 2010; Eulitz et al. 1997; Freeman 
et al. 2009). The selective cancellation can affect the relative signal-to-noise ratio (SNR) of MEG and EEG (de Jongh et al. 2005; Goldenholz et al. 2009; Huiskamp et al. 2010). Several studies have reported that epileptic spikes are sometimes detected in either EEG or MEG but not necessarily in both, even when EEG and MEG are recorded simultaneously (e.g., Knake et al. 2006; Lin et al. 2003). Spikes detected in EEG only could be due to sources corresponding to the orientation of the lowest sensitivity in MEG. Spikes that are detected only in MEG, however, cannot be easily explained by the orientation of the source alone. One reason could be spatial under-sampling due to a low number of EEG sensors. More likely, however, the SNR for these spikes is higher in MEG than in EEG because of selective cancellation of background brain noise from tangentially oriented sources, such that in EEG the spikes are obscured by radially oriented background sources (Ahlfors et al. 2010). Therefore, the presence of a source orientation to which MEG is insensitive contributes to the complementary properties of MEG and EEG beyond the case of a single focal source going undetected in MEG because it is exactly radially oriented.

Acknowledgements This work was supported by NIH grants NS057500, NS037462, and HD040712. This work was supported in part by The National Center for Research Resources (P41RR14075) and the MIND Research Network. We thank Drs. David Cohen and Maria Mody for valuable discussions and comments on the manuscript.

Open Access This article is distributed under the terms of the Creative Commons Attribution Noncommercial License which permits any noncommercial use, distribution, and reproduction in any medium, provided the original author(s) and source are credited.

\section{References}

Ahlfors SP, Han J, Lin FH, Witzel T, Belliveau JW, Hämäläinen MS, Halgren E (2010) Cancellation of EEG and MEG signals generated by extended and distributed sources. Hum Brain Mapp 31:140-149

Baillet S, Mosher JC, Leahy RM (2001) Electromagnetic brain mapping. IEEE Signal Proc Mag 18:14-30

Baule G, McFee R (1965) Theory of magnetic detection of the heart's electrical activity. J Appl Phys 36:2066-2073

Cohen D, Cuffin BN (1983) Demonstration of useful differences between magnetoencephalogram and electroencephalogram. Electroencephalogr Clin Neurophysiol 56:38-51

Cohen D, Cuffin BN (1991) EEG versus MEG localization accuracy: theory and experiment. Brain Topogr 4:95-103

Cuffin BN (1990) Effects of head shape on EEG's and MEG's. IEEE Trans Biomed Eng 37:44-52

Dale AM, Fischl B, Sereno MI (1999) Cortical surface-based analysis. I. Segmentation and surface reconstruction. NeuroImage 9:179-194

de Jongh A, de Munck JC, Goncalves SI, Ossenblok P (2005) Differences in MEG/EEG epileptic spike yields explained by regional differences in signal-to-noise ratios. J Clin Neurophysiol 22:153-158

Eulitz C, Eulitz H, Elbert T (1997) Differential outcomes from magneto- and electroencephalography for the analysis of human cognition. Neurosci Lett 227:185-188

Fischl B, Sereno MI, Dale AM (1999) Cortical surface-based analysis. II: Inflation, flattening, and a surface-based coordinate system. NeuroImage 9:195-207

Freeman WJ, Ahlfors SP, Menon V (2009) Combining fMRI with EEG and MEG in order to relate patterns of brain activity to cognition. Int J Psychophysiol 73:43-52

Fuchs M, Wagner M, Kastner J (2001) Boundary element method volume conductor models for EEG source reconstruction. Clin Neurophysiol 112:1400-1407

Geddes LA, Baker LE (1967) The specific resistance of biological materials - a compendium of data for the biomedical engineer and physiologists. Med Biol Eng 5:271-293

Goldenholz DM, Ahlfors SP, Hämäläinen MS, Sharon D, Ishitobi M, Vaina LM, Stufflebeam SM (2009) Mapping the signal-to-noiseratios of cortical sources in magnetoencephalography and electroencephalography. Hum Brain Mapp 30:1077-1086

Grynszpan F, Geselowitz DB (1973) Model studies of the magnetocardiogram. Biophys J 13:911-925

Hämäläinen MS, Sarvas J (1989) Realistic conductivity geometry model of the human head for interpretation of neuromagnetic data. IEEE Trans Biomed Eng. 36:165-171

Hämäläinen MS, Hari R, Ilmoniemi RJ, Knuutila J, Lounasmaa OV (1993) Magnetoencephalography-theory, instrumentation, and applications to noninvasive studies of the working human brain. Rev Mod Phys 65:413-497

Haueisen J, Ramon C, Czapski P, Eiselt M (1995) On the influence of volume currents and extended sources on neuromagnetic fields: a simulation study. Ann Biomed Eng 23:728-739

Haueisen J, Tuch DS, Ramon C, Schimpf PH, Wedeen VJ, George JS, Belliveau JW (2002) The influence of brain tissue anisotropy on human EEG and MEG. Neuroimage 15:159-166

Hillebrand A, Barnes GR (2002) A quantitative assessment of the sensitivity of whole-head MEG to activity in the adult human cortex. Neuroimage 16:638-650

Huang MX, Mosher JC, Leahy RM (1999) A sensor-weighted overlapping-sphere head model and exhaustive head model comparison for MEG. Phys Med Biol 44:423-440

Huang MX, Song T, Hagler DJ Jr, Podgorny I, Jousmaki V, Cui L, Gaa K, Harrington DL, Dale AM, Lee RR, Elman J, Halgren E (2007) A novel integrated MEG and EEG analysis method for dipolar sources. Neuroimage 37:731-748

Huiskamp G, Agirre-Arrizubieta Z, Leijten F (2010) Regional differences in the sensitivity of MEG for interictal spikes in epilepsy. Brain Topogr 23(2):159-164

Knake S, Halgren E, Shiraishi H, Hara K, Hamer HM, Grant PE, Carr VA, Foxe D, Camposano S, Busa E, Witzel T, Hämäläinen MS, Ahlfors SP, Bromfield EB, Black PM, Bourgeois BF, Cole AJ, Cosgrove GR, Dworetzky BA, Madsen JR, Larsson PG, Schomer DL, Thiele EA, Dale AM, Rosen BR, Stufflebeam SM (2006) The value of multichannel MEG and EEG in the presurgical evaluation of 70 epilepsy patients. Epilepsy Res 69:80-86

Lin YY, Shih YH, Hsieh JC, Yu HY, Yiu CH, Wong TT, Yeh TC, Kwan SY, Ho LT, Yen DJ, Wu ZA, Chang MS (2003) Magnetoencephalographic yield of interictal spikes in temporal lobe epilepsy. Comparison with scalp EEG recordings. Neuroimage 19:1115-1126

Liu AK, Dale AM, Belliveau JW (2002) Monte Carlo simulation studies of EEG and MEG localization accuracy. Hum Brain Mapp 16:47-62 
Marinkovic K, Cox B, Reid K, Halgren E (2004) Head position in the MEG helmet affects the sensitivity to anterior sources. Neurol Clin Neurophysiol 2004-30:1-6

Melcher JR, Cohen D (1988) Dependence of the MEG on dipole orientation in the rabbit head. Electroencephalogr Clin Neurophysiol 70:460-472

Mosher JC, Leahy RM, Lewis PS (1999) EEG and MEG: forward solutions for inverse methods. IEEE Trans Biomed Eng 46:245-259

Parkkonen L, Fujiki N, Mäkelä JP (2009) Sources of auditory brainstem responses revisited: contribution by magnetoencephalography. Hum Brain Mapp 30:1772-1782

Ramon C, Schimpf P, Haueisen J, Holmes M, Ishimaru A (2004) Role of soft bone, CSF and gray matter in EEG simulations. Brain Topogr 16:245-248

Riera JJ, Valdes PA, Tanabe K, Kawashima R (2006) A theoretical formulation of the electrophysiological inverse problem on the sphere. Phys Med Biol 51:1737-1758
Schimpf PH, Ramon C, Haueisen J (2002) Dipole models for the EEG and MEG. IEEE Trans Biomed Eng 49:409-418

Tarkiainen A, Liljestrom M, Seppä M, Salmelin R (2003) The 3D topography of MEG source localization accuracy: effects of conductor model and noise. Clin Neurophysiol 114:1977-1992

Tesche CD, Karhu J, Tissari SO (1996) Non-invasive detection of neuronal population activity in human hippocampus. Brain Res Cogn Brain Res 4:39-47

Wolters CH, Anwander A, Tricoche X, Weinstein D, Koch MA, MacLeod RS (2006) Influence of tissue conductivity anisotropy on EEG/MEG field and return current computation in a realistic head model: a simulation and visualization study using highresolution finite element modeling. Neuroimage 30:813-826

Yvert B, Bertrand O, Echallier JF, Pernier J (1996) Improved dipole localization using local mesh refinement of realistic head geometries: an EEG simulation study. Electroencephalogr Clin Neurophysiol 99:79-89 